\title{
ORDEN TUTELAR Y EFICACIA MORAL. CONSIDERACIONES SOBRE LA DESTRUCCIÓN Y LA INVENCIÓN $^{1}$
}

\author{
Guillermo Nugent ${ }^{2}$
}

\begin{abstract}
Resumen / Abstract
El artículo sostiene la tesis que la eficacia moral de la Iglesia Católica en Latinoamérica se juega en la salvación a nivel comunitario y no en la responsabilidad individual, manifestada en la brecha entre moral ceremonial y moral cotidiana. La alternativa a dicha autoridad tutelar no parece ser su reemplazo por el deber universal de la razón, más bien consiste en el reconocimiento de una moral de la invención- La eficacia moral pareciera surgir, más bien, de una combinación de ideas, emociones, estilos, hábitos, que efectivamente regulen las interacciones en la vida social. En esa línea, se plantea que a partir de los acontecimientos que han marcado los últimos treinta años en la vida pública peruana (aunque se trata de una tendencia extendida a escala global), comienza a aparecer una moral que toma como extremos la destrucción y la invención. Su trasfondo es la confianza básica colectiva que permite ensayar la satisfacción de necesidades y deseos a través de la creatividad.
\end{abstract}

Palabras clave: moral tutelar, Iglesia Católica, ceremonia, moral individual, nueva moral

\section{TUTELAR ORDER AND MORAL EFFICIENCY. CONSIDERATIONS ABOUT DESTRUCTION AND INVENTION.}

The article makes the claim that the moral efficacy of the Catholic Church in Latin America is played in salvation at the community level and not on individual responsibility, whatever manifested in the gap between ceremonial moral and everyday moral. The alternative to such supervisory authority does not seem to be his replacement by the universal duty of reason, rather it consists in the recognition of a moral of the invention. The moral effectiveness appear

1 Este trabajo se benefició de manera apreciable con las observaciones de Ana Nugent y Juan Luis Nugent, mis hijos, así como de mi apreciada colega Marcela Benites.

2 Universidad Nacional Mayor de San Marcos. Facultad de Ciencias Sociales. E-mail: gnugent@ unmsm.edu.pe 
to arise from a combination of ideas, emotions, styles, habits, that effectively regulate interactions in social life. In that vein, it is argued that from the events that have marked the last thirty years in Peruvian public life (although it is a widespread tendency to global scale), starts to appearing a morality that takes as bounds destruction and the invention. His background is the collective basic trust that allows testing the satisfaction of needs and desires through creativity.

Keywords: tutorial moral, Catholic Church, ceremony, individual morality, new moral

\section{Moral ceremonial y moral cotidiana}

La eficacia moral es en cierta medida una redundancia: una moral que no fuera eficaz perdería su razón de ser. ¿Cómo evaluar esa eficacia, en qué áreas de la vida social encontrarla? Esta es una pregunta básica para empezar una exploración sobre alternativas a los discursos morales que emanan del orden tutelar. ${ }^{3}$ Por una parte, tenemos los enunciados morales cuya principal función aparentemente consiste en quedarse como enunciados y en otra dimensión tenemos las costumbres. Muy rara vez se menciona la costumbre como la fuente de unas prácticas que merecen ser imitadas o seguidas por otros. Por lo general los ideales son formulados como enunciados antes que como un conjunto de acciones que son consideradas en algún sentido ejemplares y que merecen el esfuerzo de ser imitadas. Las costumbres, especialmente en países que tuvieron sociedades originarias altamente organizadas, tienden a ser asociadas a todo lo que no es civilizado, incapaz de ser articulado como enunciado. No obstante, ninguna moral adquiere vigencia en virtud de sus enunciados. La dimensión práctica ideal está encarnada en las ceremonias. En la mejor situación, la ceremonia cumple una función de síntesis del conjunto de experiencias sociales, incluidos los objetos que intervienen en esos momentos, además de los personajes. En el otro extremo, la ceremonia puede funcionar como una especie de compensación de las frustraciones de la vida diaria.

Se trata entonces de distinguir una moral ceremonial, de una moral cotidiana. La primera es puesta de manifiesto en circunstancias ritualizadas, que van desde los cumpleaños hasta los entierros, pasando por las procesiones, inauguraciones, desfiles y todo lo que usualmente consideramos como un espacio y un tiempo protocolar. Generalmente la sanción por no participar de estos momentos suele estar asociado con algún grado de vergüenza.

3 En este trabajo desarrollo algunos planteamientos que fueron expuestos en Nugent 2010. 
La segunda es la que tiene que ver con lo que podemos llamar momentos más bien profanos, aquellos que no guardan relación con un momento comunitario. Su razón de ser es ordenar el desenvolvimiento de la individualidad en el mundo de las interacciones tanto en espacios públicos como privados. Las infracciones a este tipo de normas más allá de un cierto umbral se suelen llamar delitos. Cuando se es afectado por el incumplimiento de estas normas surge con cierta nitidez el sentimiento y la convicción de estar ante una injusticia. Por supuesto, pueden haber normas injustas, que no merecen ser acatadas, pero en este caso se trata de situaciones permanentes que no producen una disrupción con las reglas de juego pues la injusticia está en las propias reglas. A veces puede ser un claro abuso o discriminación que no está tipificado como delitos formales y que suscita el inmediato sentimiento de estar ante una injusticia y que puede dar lugar a reclamos o protestas que pueden asumir expresiones individuales o colectivas. Ambas dimensiones, la ceremonia y el conjunto de acciones cotidianas, son fundamentales para reforzar un sentido de comunidad y de identidad individual.

En muchos países latinoamericanos hay un escenario donde las dimensiones morales que se refieren a un sentido de protección y delimitación de la individualidad están en una situación más bien precaria: asaltos, secuestros, homicidios y delitos similares tienen una tasa de incidencia notoriamente alta en la región. Sin embargo, la atención a los momentos ceremoniales no parece haber tenido un declive significativo.

Este es el punto de partida entonces: un escenario donde la moral ceremonial ocupa un lugar prominente en los espacios públicos y una moral cotidiana cuya precariedad es cada vez más notoria y sobre todo menos tolerable. ¿ Cómo podemos imaginar alternativas a esta situación? El problema es que en los discursos públicos, la cohesión moral de la colectividad se hace casi exclusivamente en términos de la moral ceremonial. En América latina, con pocas excepciones, encontramos una significativa brecha entre la moral ceremonial y la moral cotidiana.

En esta configuración de las cosas corresponde preguntarnos ęcuál es la función pública de la moral religiosa y en especial la católica; en qué áreas posee eficacia en cuáles no? Es una verdad aceptada que el continente latinoamericano es 'muy religioso' - una afirmación básicamente política- y a la vez es una de las regiones del mundo donde aparte de las extremas desigualdades, hay una incidencia sin equivalente de las violencias callejeras, en el área de lo que se llama los delitos comunes. Por supuesto, las quejas sobre la corrupción de las autoridades son un trasfondo constante en las discusiones públicas. Es decir, el discurso religioso no parece tener una especial influencia para 
atenuar o suprimir las acciones delictivas, salvo a posteriori. La importancia del arrepentimiento es un momento moral especialmente dramático. El caso tan frecuente del temible delincuente que se convierte en predicador religioso durante el tiempo de la prisión. Es decir, una religiosidad que debe ser entendida como un marcado sentido de comunidad que coexiste con una notoria incapacidad para asegurar la convivencia pacífica de las personas en su vida diaria. ¿Cómo entender este salto de la ceremonia en la que todo el mundo participa para reafirmar objetivos comunes con una vida diaria donde justamente la referencia a todos los demás está muy ausente y en cuya atmósfera la desconfianza parece permear todo?

Montaigne señalaba que el fundamento místico de la autoridad consistía en obedecer a las leyes...porque son leyes ${ }^{4}$. Con ello hacía un llamado a reconocer la importancia de las experiencias individuales para el propio conocimiento y la comunicación de las personas ${ }^{5}$. Lo aprendido en la experiencia era más importante que el halo de autoridad que emanaba de las leyes.

Dada la enorme influencia de la iglesia católica en los países latinoamericanos es significativa su muy baja incidencia en la regulación moral de las conductas individuales, no obstante el magnetismo desplegado en la convocatoria de muchedumbres en procesiones y fiestas religiosas. Este es un problema de importancia central para entender por qué es importante una moral laica, precisamente en el ámbito de las interacciones cotidianas.

Podría suponerse que el catolicismo aspira únicamente a la identificación colectiva, sin ocuparse necesariamente del ámbito individual, como si estuviera, por ejemplo, en una situación paralela a las ceremonias castrenses. Ambos se moverían en el terreno de las interpelaciones puramente comunitarias. Se trata de una apreciación equivocada. Si asumiéramos esta perspectiva no se podría entender el vivo interés de la iglesia católica en mantener una influencia en espacios que no son ceremoniales a través de los cursos de religión en las escuelas públicas, como sucede en la mayoría de los países latinoamericanos, en otras palabras, una preocupación por la trasmisión de un discurso en un espacio que no es ceremonial.

4 "Ahora bien, las leyes mantienen su crédito no porque sean justas, sino porque son leyes. Este es el fundamento místico de su autoridad; no tienen otro. Lo cual les conviene mucho. A menudo están hechas por necios, las más de las veces por gente que, por odio a la igualdad, carece de equidad, pero siempre por hombres, autores vanos e inciertos." Agrega, en referencia las leyes francesas de su tiempo: "El mandato es tan confuso e incierto, que en cierto modo excusa la desobediencia" (Montaigne 2007 pp.1601-1602). Jacques Derrida dedicó un trabajo a este tema, pero en una perspectiva diferente a la tratada aquí (Derrida et al. 1997).

5 "Cuando la razón nos falla empleamos la experiencia" (Montaigne 2007. p.1589). 
Más nítido todavía es el contraste con la intransigente participación que tiene en asuntos que característicamente corresponden a conductas individuales, más bien íntimas, como son las prácticas de la sexualidad. Queda claro entonces que las pretensiones de la moral católica no se agotan en la invocación comunitaria, como podría ser por ejemplo, la de 'pueblo de Dios'. Le interesa vivamente regular el comportamiento individual de los individuos... aparentemente.

\section{Herencias y procesiones}

¿Cómo entender la eficacia moral en este contexto? O quizás corresponde indagar qué aspecto en particular de la regulación de la sexualidad es el que resulta más relevante? La respuesta es fácil: el vínculo matrimonial, por ser el que históricamente definía la legitimidad de los patrimonios y en consecuencia de las herencias. El interés por la regulación de la sexualidad mediante la autoridad para dar legitimidad a los matrimonios, está a la base del gran poder político que adquirió la iglesia en tierras europeas desde hace al menos un milenio ${ }^{6}$. La aparición de estados laicos después de la revolución francesa y sobre todo la lenta pero sostenida difusión en los siglos siguientes de la administración estatal de los registros civiles (nacimiento, matrimonio, defunción), además de la administración estatal de los cementerios, pusieron claros límites a la prerrogativa eclesiástica para determinar la legitimidad de los vínculos de parentesco ${ }^{7}$. Podemos entender, entonces, la marcada susceptibilidad de la institución frente a cualquier modificación que surja en el ámbito del parentesco y la reproducción y por extensión en el ejercicio de la sexualidad en general.

Esta situación sin embargo se ha desplegado en nuestros países con una notoria debilidad en asegurar la legitimidad de los vínculos conyugales fuera del espacio de las élites políticas y económicas. De ahí que la capacidad regulatoria de la sexualidad del catolicismo no haya sido particularmente eficaz si consideramos la sociedad como un todo. Pero sí fue lo suficientemente cercana a los ámbitos del poder como para poder asegurar una posición

6 Duby (1982). Puedo agregar que según los documentos de la Inquisición en el Archivo Nacional de México, los primeros procesos son por el delito de 'amancebamiento'. Es decir, como los jefes aztecas no estaban casados por la religión católica estaban en falta y la sanción más frecuente era la pérdida del patrimonio. La Inquisición en la práctica estuvo 'más interesada en la apropiación de patrimonios que en la persecución de herejías. Véase también el amplio estudio de Brundage (2000).

7 Un trabajo reciente para el caso del Perú, con énfasis en las discusiones que generó el matrimonio civil, es el deVillanueva Salvatierra 2013, pp. 183-226. 
preferencial en el discurso estatal. Se puede entender así la figura, en apariencia paradójica, que por un lado muestra el énfasis puesto en mantener un cierto orden legal y, por otro, una laxitud en cuanto a su vigencia: legalidad para la élite y ceremonia para las masas. Podemos afirmar, entonces, que hubo y hay dos niveles bastante diferenciados del ejercicio de la presencia católica en la vida pública. El primero, en su cercanía al ejercicio de las funciones de gobierno, prestando su capacidad legitimadora para las acciones políticas, y el segundo, en un nivel ciudadano más amplio, a partir de las ceremonias públicas como las fiestas religiosas y las procesiones.

El reto que enfrenta la iglesia católica en América Latina por lo tanto ocurre en dos frentes: en el estado donde su gran adversario es la presencia de una cultura secular en expansión no solamente en las élites sino también en el ejercicio de la función estatal como tal; y a nivel de la ciudadanía la competencia planteada por las iglesias cristianas, en especial evangélicas y los medios de comunicación. En el caso de los medios, no se trata de una competencia religiosa; ocurre que hay un formato que es crecientemente participativo y que desdibuja el tiempo ceremonial jerárquico. En un cierto sentido los santos son reemplazados por las estrellas del mundo del espectáculo. A diferencia de las iglesias cristianas, aquí no se trata de una pugna en torno a la verdad de algunas afirmaciones como de la alteración del espacio, del escenario para la comunicación.

\section{¿Y Cómo funciona la moral católica en la vida diaria?}

Pero vale la pena hacer la pregunta ¿̇cuál es el nivel de eficacia de las normas de la iglesia católica para el manejo de los asuntos en la vida diaria? La pertinencia de la pregunta queda subrayada si recordamos que la región, aparte de tener los más pronunciados índices de desigualdad a escala global, posee también las mayores tasas de criminalidad callejera. Alguna eficacia deben tener esas normas, de lo contrario el peso público de la institución religiosa sería apenas perceptible. Mientras que la desigualdad se puede plantear en términos 'estructurales' es decir haciendo abstracción de acciones individuales particularizadas, no es el caso con la criminalidad callejera y la corrupción.

Este proceso, el de la criminalidad, corresponde a una multitud de hechos claramente individualizables y que se ubican en el terreno de las acciones morales. Como es fácil observar, ya no está en juego únicamente un asunto de políticas públicas redistributivas para atenuar o disminuir de manera radical, según sea el caso, la desigualdad. La criminalidad nos coloca de lleno en el terreno de los límites morales de las acciones individuales. La mayor visibilidad de la criminalidad coloca, en consecuencia, también en primer plano las reglas 
y los estilos de moralidad que forman parte de las creencias de las personas. La parte más visible de los debates públicos se refieren a medidas policiales y judiciales para limitar las múltiples formas de delincuencia. Están en cuestión los criterios morales de la vida cotidiana que hasta ahora han tenido vigencia y sobre este aspecto es relativamente poco lo avanzado. La eficacia moral de las instituciones clásicamente tutelares está seriamente cuestionada.

¿Esa eficacia está en el terreno del orden público, del respaldo a un sentido de legalidad, en particular de un sentido de igualdad ante la ley? No, por supuesto. ¿Dónde se instala la eficacia moral de la iglesia católica entonces? En una tarea de legitimación, tutelar, del estado y la correspondiente devolución de favores institucionales. Hay una noción de pueblo a ser salvado, donde la salvación se juega a nivel comunitario y la responsabilidad individual en esa tarea es francamente tenue por decir lo menos. La eficacia gira en torno a la salvación comunitaria y queda graficado en el mundo de las ceremonias. La intensidad de las emociones colectivas pareciera superponerse a la serie de orientaciones cotidianas que marcan la vida diaria. Es un tiempo ceremonial y es ahí donde despliega su poder. ${ }^{8}$

Tal es la importancia de las ceremonias donde lo que importa es la cohesión del todo antes que nada. Esto explica la poca preocupación del clero por el cumplimiento individual efectivo de sus normas, en materia de sexualidad por ejemplo, pero el tremendo celo institucional en lo que a promulgación de leyes referidas al ejercicio de la sexualidad. Si los cursos obligatorios de religión en las escuelas públicas tienen una eficacia práctica que sirva como referencia en los asuntos diarios de los jóvenes es una cuestión supletoria. Lo que importa es señalar en cuanta ocasión sea posible, cómo el orden público tiene como garante no tanto a la legalidad democrática como a instituciones que ofrecen una custodia del todo, una de ésas es justamente la iglesia católica. El socorro a los pobres, los incapacitados de representarse a sí mismos, adquiere una importancia central porque en ese ejercicio de la caridad, que deja en impagable deuda de agradecimiento a sus beneficiarios, el retorno, la devolución, consiste en la obediencia a la institución en su carácter tutelar, antes que en la orientación cotidiana en los asuntos prácticos. ${ }^{9}$

Se entiende mejor entonces, en ese estado de cosas, el carácter de

8 Canetti (1982, p. 153) mencionaba a la lentitud como un recurso para el ejercicio del poder por parte del catolicismo romano: "En este mundo nada goza del inmovilismo de los bienaventurados: sin embargo, cualquier cosa que muestre la iglesia ha de mostrarlo lentamente. Las procesiones son un ejemplo impresionante."

9 Es importante tener presente la observación de Simmel sobre el pobre como aquel que es definido por el socorro que recibe, antes que por las carencias. 
ofensa intolerable que tiene cualquier defensa de la laicidad del estado. Es una amenaza al pacto tutelar, el que sostiene que la mejor forma de gobierno es una protección benefactora antes que un reconocimiento de derechos, es decir la cooperación ciudadana. Hay una cierta ironía cruel en este orden de cosas, pues al haber una laxitud en el cumplimiento de normas lo que se instaura es una atmósfera de desprotección legal generalizada. Este aspecto no ha sido suficientemente subrayado en las discusiones sobre el tema: en efecto, la cuestión no es solamente que hay un relajamiento en seguir las normas, esto en sí mismo no tendría porqué ser negativo necesariamente, pueden haber normas injustas, otras, en cambio, pueden quedar abiertas a consideraciones contextuales.

La contraparte es que esa laxitud se traduce en un clima de desprotección legal generalizado que reclama una solución de tipo tutelar, es decir inhibirse de ejercer la representación. En ese estado de minusvalía ciudadana es que se asientan las raíces más firmes de un ejercicio tutelar de la autoridad, una de cuyas vertientes es el clericalismo.

Esta solución es efectiva en cuanto a sus fines tutelares pero de muy limitado alcance para respaldar los derechos individuales. Aquí está en juego la cuestión de la laicidad. En sentido estricto, la laicidad en América Latina no se puede reducir a la separación entre iglesia y estado. Es sobre todo una liberación del ejercicio de la autoridad tutelar y la creación de una comunidad menos afectada, más diversa, sin uniformes o sotanas y con un tiempo ceremonial donde la sencillez y la solemnidad permiten crear genuinos sentimientos igualitarios en medio de la diversidad de individualidades.

Nunca será suficiente insistir en la predilección del ejercicio tutelar de la autoridad por el tiempo ceremonial rígidamente jerarquizado donde el todo social, por así decir, queda condensado en una gran cadena de subordinaciones. En la práctica, en la economía de las emociones, la ceremonia se supone que debe cumplir la función reguladora que corresponde a las leyes. En esta parte me parece necesario llamar la atención sobre la virtual escisión entre oralidad y escritura que se instaló con especial fuerza en aquellos territorios con sociedades originarias en los tiempos de la colonización y que se convirtió en una estrategia política de los estados republicanos: la posesión del papel como símbolo de la autoridad ${ }^{10}$. La ceremonia es un medio de comunicación cuya eficacia tiende a estar en proporción inversa a la difusión de la palabra impresa. Surge la cuestión de en qué medida la difusión de la cultura audiovisual de base eléctrica y digital ha modificado el significado del momento ceremonial.

10 Una aproximación inicial fue planteada en Nugent (1996). Véase también el trabajo de Juan Biondi y Eduardo Zapata (2006) que explora ampliamente esta cuestión. 
Es una línea de investigación que encuentro impostergable.

\section{¿La moral es una cuestión de estilo?}

¿Cuál puede ser entonces la alternativa? ¿Consistirá acaso en recurrir al fundamentalismo de la letra? Es decir en cumplir al pie de la letra la escritura sagrada o los textos legales en los comportamientos civiles. La eficacia de este recurso está comprobada en grupos pequeños, pero adolece de muy limitada elasticidad para regular amplios grupos humanos.

En América Latina el rigorismo no ha sido una pieza del repertorio disponible de la imaginación moral colectiva. Por el contrario, estas tierras aparecen en el canon occidental como el mundo del goce, de los disfrutes relajados por oposición al deber universal ${ }^{11}$. No parece que reemplazar el tutelaje por el deber universal de la razón guarde relación con el estilo y las costumbres que permiten la participación en una comunidad. Esta observación merece ser tenida en cuenta no solamente en cuanto al rigorismo acerca de la observancia de normas religiosas, también alude a ciertas maneras de entender los procesos de democratización.

El estilo moral es una dimensión muy raramente problematizada pero de importancia capital para calibrar las transformaciones en el orden de las costumbres ¿y cuál es ese estilo? Es algo que se mueve en el espectro que va desde la picaresca plebeya de un Cantinflas, pasando por los desenfadados que abundan en las páginas de Jorge Amado hasta la ironía burlona de $\mathrm{H}$. Bustos Domecq, el personaje inventado por Borges y Bioy. Esos estilos son a las comunidades como los rasgos de carácter a los individuos: algunas cosas se pueden cambiar pero la manera de hacerlas, los gestos, son algo muy propio...y muy práctico. Los estilos no necesitan ser pensados, puede ocurrir que sean la consecuencia de un cierto conjunto de ideas y creencias, así como de ciertos episodios históricos o también pueden ser la base 'para una posterior elaboración moral.

Probablemente el mayor error a este respecto ha sido querer hacer una

11 Kant, al discutir la relación entre la ley universal y los deberes hace esta interesante observación, que rara vez, si alguna, ha recibido atención por parte de filósofos de 'los mares del Sur: "Entonces advierte que sin duda la naturaleza siempre puede subsistir con arreglo a semejante ley universal, aun cuando el hombre (tal como hacen quienes habita los mares del sur) deje enmohecer su talento y consagre su vida simplemente a la ociosidad, la diversión y la procreación, en una palabra, al goce" (Kant, 2002, p. 107). 
teoría como desprendida de cualquier estilo, y esto es particularmente decisivo a propósito de una cuestión como la autoridad. Es cierto que no pocas veces se ha establecido la relación entre una doctrina o conjunto de ideas y el carácter de su autor, pero aquí me refiero al razonamiento moral que forma parte de un cierto estilo cultural. Si podemos reconocer y aceptar ese estilo entonces habrá más claridad sobre lo que vale la pena mantener y aquello que no resulta imprescindible.

El estilo al que aludo es, en los mejores casos, una curiosa instancia donde puede reconocerse tanto la calidez como la resignación. Ésta, sin embargo, en no pocas ocasiones encubre una especie de rabia social ante las humillaciones y desprecios padecidos en carne propia o a través de los relatos de los progenitores. Quizás el racionalismo surge de la convicción, o siquiera la esperanza de poder controlar y ordenar la realidad con los recursos de la razón, de abstracciones. La peculiaridad de las mejores filosofías y creaciones artísticas es que simultáneamente traducen maneras existentes de abordar las dificultades de la existencia, sin excluir las emociones. Diferente es el caso de las normas a implementar desde una autoridad previa, sea una religión escrita (porque está escrita tiene normas aplicables en principio en cualquier lugar y época) o un conjunto de leyes del estado o, con más frecuencia, de un estado religioso.

La eficacia moral, consiste entonces, en un conjunto de planteamientos, una combinación de ideas, emociones, estilos, hábitos, que efectivamente regulan las interacciones en la vida social. ${ }^{12}$ Hay zonas de compatibilidad y de incompatibilidad moral que es importante tratar de distinguir. En los estados latinoamericanos, en la mayoría de ellos una relación estrecha con la iglesia católica es considerado un componente central para la legitimidad del orden público.

12 Un ejemplo: en la tercera semana de marzo del 2013 en Lima hubo dos marchas que tuvieron una cierta cobertura noticiosa: el sábado fue una marcha 'por la vida', en víspera de una fecha conmemorativa de los derechos del no nacido, es decir, una marcha contra cualquier intento de legalizar o siquiera despenalizar el aborto. No dejaba de llamar la atención la convocatoria de la marcha pues en esos momentos no se estaba discutiendo un proyecto de ley ni había algún grupo de presión que estuviera proponiendo un dispositivo legal en ese sentido. Al día siguiente, hubo otra marcha, más concurrida, también por la vida, pero en este caso referida a la integridad física de las personas y la inseguridad correspondiente tanto en la calle como en sus viviendas o centros de trabajo. Pues bien, en esta segunda marcha no estuvo presente ninguna autoridad católica particularmente notoria; en la anterior, como es previsible, participó el cardenal de Lima. El mensaje era claro: los problemas de inseguridad ciudadana son un problema menor desde el punto de vista de la salvación, donde la defensa de los derechos de la condición fetal ocupan el primer lugar. (http://www. larepublica.pe/23-03-2013/manana-se-espera-gran-acogida-en-la-marcha-por-la-seguridad-delperu). 


\section{El difícil arrepentimiento secular}

El aspecto a confrontar es el siguiente: primero, preguntarse ¿cuáles son los principales problemas de moral cotidiana? La violencia callejera y la corrupción en el manejo de los asuntos públicos suele ser un dato estable en la mayoría de países del continente. Los grados pueden variar considerablemente de un país a otro, pero siempre se trata de una diferencia de grados dentro de un mismo espectro. Dejamos por el momento fuera de estas consideraciones el caso de las dictaduras militares que impulsaron políticas deliberadamente crueles (torturas, asesinatos, desapariciones) que tuvieron una relación más o menos estrecha con la iglesia católica ${ }^{13}$. Me interesa ocuparme más bien de los escenarios más cotidianos. Un elemento que siempre ha acompañado al catolicismo es la compatibilidad moral que establece entre religión y delincuencia. Es decir, los grandes delincuentes en no pocas ocasiones suelen ser personas de ostensibles gestos de catolicidad, muestran un lado religioso que es compatible con acciones moralmente censurables desde el pùnto de vista público que, por lo general aparece como la distinción entre delito y pecado, para atenuar el primero, claro. Como ya señalamos anteriormente esta zona de compatibilidad implica una tensión entre salvación y justicia secular. Aquí también tenemos una discusión pendiente en nuestros propios vocabularios culturales.

Inversamente, hay zonas de incompatibilidad por ejemplo en lo que se refiere a todo lo que es regulación de la sexualidad, históricamente la base del poder político de la iglesia católica, el divorcio civil y la extensión del matrimonio a parejas del mismo sexo, la legalización del aborto, la oposición al uso de preservativos incluso en contextos de presencia de enfermedades de transmisión sexual. En estos casos, la incompatibilidad es a un nivel institucional, pues no es plausible asumir que tales normas de la conducta íntima sean necesariamente seguidas por quienes participan de las ceremonias religiosas del catolicismo.

Nos encontramos entonces con un problema crítico. Las prácticas que generan preocupación cuando no indignación ciudadana: la criminalidad callejera, la corrupción y en general el conjunto de deshonestidades en la vida pública y que muchas veces están sancionadas penalmente, se mueven en una zona moralmente compatible para los delincuentes, que no ven en la comisión del delito un obstáculo para la salvación. Esta situación es una consecuencia

13 Dado que el papa actual, Francisco, es argentino, las obras de Horacio Verbitsky sobre la iglesia católica en Argentina adquieren una especial relevancia (Verbitsky 2006 y 2007). 
perversa del énfasis clerical en la normatividad sexual como el límite entre la salvación y la condena. A fuerza de hacer hincapié en este aspecto, el resto de prácticas transgresoras adquiere un inevitable tono menor. La legalización del matrimonio también para las personas homosexuales desata iras y amenazas clericales que rara vez se escuchan contra las mafias del narcotráfico o los asesinatos, asaltos, estafas. El mensaje implícito es que la criminalidad en sus distintas formas es una cuestión menor en comparación con la legalización del divorcio civil, antes y ahora, con cuestiones tales como la la legalización del aborto o ampliar el derecho al matrimonio civil. Este énfasis unilateral en la reglamentación de la sexualidad se ha traducido en una actitud bastante laxa frente a las cuestiones de la inseguridad ciudadana .

¿Cómo abordar entonces la cuestión de establecer un conjunto de normas prácticas, algunas legales, otras simplemente rutinarias, las más importantes, que permitan establecer una suficiente eficacia moral?

La cuestión de la inseguridad ciudadana, es un problema bastante preocupante en varias ciudades capitales de América Latina. Sea por el narcotráfico, por bandas de secuestradores, asaltos callejeros, hay una preocupación pública por ser víctima en cualquier momento de un asalto u objeto de un asesinato, para no mencionar otras precariedades que amenazan la vida diaria como los accidentes de tránsito ocasionados por choferes absolutamente irresponsables, que suelen llevar rosarios e imágenes religiosas colgadas del espejo retrovisor. Justamente por este tipo de situaciones es que los delitos suelen ser consideradas faltas morales leves. Es un caso donde las demandas de la salvación y la de la justicia secular tienen prioridades muy diferentes. Esos episodios muestran que hay una dificultad seria de moral pública: la salvación y los discursos correspondientes tiene pocas áreas de coincidencia con las necesidades de la justicia secular. No se trata de una escisión completa, pero es claro que la preocupación por la salvación religiosa no hace a la gente mejores ciudadanos necesariamente.

\section{La destrucción y la invención. Una nueva moralidad}

A partir de los acontecimientos que han marcado los últimos treinta años en la vida pública peruana, (aunque se trata de una tendencia muy extendida que hoy la podemos encontrar a escala global) me permito proponer un razonamiento moral que toma como extremos la destrucción y la invención. Sostengo que estas dos cualidades de las acciones ha sido lo más característico de nuestra vida pública reciente. De una parte, la violencia política donde el terrorismo de Sendero Luminoso estuvo orientado preferentemente hacia la 
destrucción en todas sus formas. No se trató, y es un aspecto fundamental, de una destrucción fría de autómatas aislados del resto de la sociedad, al estilo de las matanzas perpetradas por asesinos solitarios en EEUU. Por el contrario, nos encontramos frente a una destrucción apasionada, guiada por la capacidad de generar miedo. Como resulta obvio, no se destruían cosas o asesinaban a personas como incidencias aisladas, era una atmósfera de destrucción más que de terror únicamente. Fue un período de una violencia extrema donde la destrucción fue el elemento dominante. precisamente este aspecto es uno de los rasgos diferenciales del tipo de violencia que hubo aquí respecto de otros movimientos políticos que desarrollaron acciones armadas contra sus respectivos estados. La destrucción en esos casos en buena cuenta eran formas de sabotaje, que son propias de cualquier acción bélica. Pero en el caso nuestro nadie o casi nadie tipificaría las acciones de destrucción perpetradas por Sendero Luminoso como simple sabotaje. Eran más bien unas cartas de presentación moral, por así decir: había llegado el tiempo de la destrucción. Ese era el anuncio, la propuesta de una moral de la destrucción. La espectacularidad versus las desapariciones.

Simultáneamente, aparecían en el mismo escenario del día a día distintas formas de invención que la sociología determinista calificó como 'estrategias de supervivencia' pero no se trataba ni únicamente o principalmente de un movimiento reactivo. Lo que en un momento se llamó economía informal, el crecimiento urbano o el actual reconocimiento de la comida peruana fueron expresiones de una nueva actitud ante el entorno. Era una moral de la invención donde el ingenio y la adaptación fueron los principales soportes de la creatividad, una ilustración práctica de los principios sostenidos por el filósofo inglés Alfred Whitehead (1861-1947). ${ }^{14}$

¿Cuáles eran los criterios básicos de esa moral de la invención? Uno de ellos y de importancia central es 'todo sirve' cualquier elemento puede ser desprendido de un conjunto previo y ser utilizado para crear otro conjunto. Una práctica que no se limita a lo que ahora se llama reciclamiento.

Este importantísimo proceso ha pasado desapercibido en primer lugar por su carácter plebeyo, por así decir, no surgido de las universidades, de los grupos especializados en la producción de saberes; en segundo lugar, tampoco hubo un interés académico en tratar de entender las transformaciones en el mundo social desde el punto de vista de la invención, de la novedad. Por el contrario la tendencia dominante en los trabajos académicos ha sido el pasadismo. El querer interpretar todo el presente como si fuera un precipitado

14 Whitehead (1944). 
pasivo del pasado. Esto fue especialmente notorio en en el período de la más extrema violencia política donde abundaban explicaciones que pretendían establecer un nexo causal con acontecimientos de varios siglos antes, el recurso favorito era 'la herencia colonial'

Me interesa destacar que este conjunto de practicas y de objetos ha creado una particular atmósfera moral, un campo donde la oposición principal es entre destrucción e invención y todo indica que se trata de una oposición donde ambos polos van a estar presentes por un buen tiempo. Son las marcas que han quedado de un proceso particularmente conflictivo y sangriento de nuestra historia reciente. Así como las tendencias hacia la destrucción se mantienen, y que bien puede ser considerada una expresión de la pereza de construir nuevos mundos, el impulso de la invención se manifiesta en una especie de autoconfianza todavía instintiva, sin tener un adecuado reconocimiento institucional; probablemente esta sea una tarea inmediata de los tiempos venideros.

'Todo sirve' antes que nada quiere decir que tenemos a nuestra disposición la realidad para encontrar en ella los recursos para darle forma a la satisfacción de necesidades con prescindencia de un modelo previamente reconocido. Esto produce un efecto característico: las invenciones aparecen como chocantes cuando son vistas desde el mundo establecido. Ya se trate del uso del lenguaje, de la música, los colores, la arquitectura y un largo etc. Es decir, hay un trasfondo de confianza básica colectiva que permite ensayar la satisfacción de necesidades y deseos a través de invenciones ${ }^{15}$.

El ingenio aparece como la más efectiva superación del resentimiento y sin necesidad de echar mano de ningún tipo de moralismo. El soporte de esta moral de la invención radica justamente en el ingenio, en la capacidad de construir nuevas cosas, vínculos, muchas veces sin modelos ni métodos previos. El ingenio no lo es todo pero ciertamente es el primer paso indispensable para situarse ante la realidad en la perspectiva de la invención y la novedad.

15 La crisis en el pensamiento de buena parte de las ciencias sociales justamente ha consistido en eso: esforzarse en mirar el pasado estáticamente para evitar complejizar las explicaciones del presente. Sociólogos, antropólogos e historiadores se convirtieron en estatuas de sal, como le ocurrió a la mujer de Lot que miró hacia atrás, justamente para ver la destrucción de Sodoma y Gomorra, en el relato del Génesis. Mientras ello ocurría el esfuerzo de moverse en la dureza diaria para mejorar las condiciones de vida no fue algo que valiera la pena de ser observado y reconocido. La creación es una forma de olvidar el pasado y renovar tradiciones, estilos. A veces esos procesos no necesariamente pasan por la creación ex-nihilo. Pueden tomar, y con frecuencia ha sido el caso, elementos de otros conjuntos y adaptarlos a las prácticas existentes. Este es un vastísimo campo para la investigación que se ha abierto en las décadas recientes y que apenas ha sido explorado. Apenas si ha sido mencionado para seguir la trayectoria de preferencias políticas en momentos de elecciones. 
Para entender la realidad como un proceso de incesantes transformaciones, de acontecimientos que pueden relacionarse entre sí de maneras múltiples, el ingenio es indispensable. Así es como pasamos de entender la realidad como parte de un universo rígido a reconocer que estamos en un plano de multiuniversos, para recoger una expresión de William James.

Si lo anterior es aceptado entonces una moral de la amenaza y el tutelaje pierden el peso que tradicionalmente han tenido y surge un horizonte donde nuevas formas de sociabilidad y de reconocimiento del yo empiezan a estar presentes. Este desplazamiento está entre los más importantes que han tenido lugar en nuestra vida pública. El centralismo administrativo en las decisiones políticas en cierta forma ha sido una forma de prolongar esa cultura del tutelaje, por cierto.

¿Cómo plasmar un nuevo sentido de eficacia moral que recoja estas innovaciones de las décadas recientes? Quiero enfatizar que no se trata de cambios de poca monta, son tan decisivos como otros que son más fácilmente reconocibles a escala global, como la creciente autonomía de las mujeres, una nueva conciencia ecológica de preservación del medio ambiente, una muy visible valoración de la paz por sobre la guerra, y una mayor sensibilidad para la vigencia de los derechos humanos.

Podemos considerar entonces que las faltas morales como la corrupción y el crimen son formas de destrucción. Destrucción del trabajo honrado y sobre todo bien hecho, de la convivencia pacífica, de la confianza ciudadana y fundamentalmente, destrucción de vidas humanas. En nuestras culturas la corrupción y el crimen son diferentes caminos para ahogar la creatividad y en consecuencia promover la reproducción del tutelaje. Por el contrario, el orgullo por el trabajo bien hecho ${ }^{16}$, el despliegue afortunado del ingenio, la 'potencia de obrar' al decir de Spinoza, es la base más sólida para la superación del Orden Tutelar .

La eficacia moral no debe ser confundida con la eficacia penal, como sucede con frecuencia. Se la debe entender, a la eficacia moral, como un tipo de propuesta que, arraigada en las prácticas ya existentes, rutinarias, goza de credibilidad y sin mayor contacto o áreas de compatibilidad con hechos condenables por la justicia secular. El mundo de las ceremonias, religiosas y castrenses, no desaparece pero pierden el carácter de fundamento de las virtudes cívicas. En lo que a la eficacia penal se refiere, la gente puede pedir castigos

16 Este aspecto de la satisfacción por el trabajo bien hecho como gratificación básica del yo es elaborado con detalle por Richard Sennett (2009). 
más duros como solución al crimen y la corrupción, pero sin una moral de la invención como soporte, hay motivos para pensar en lo inconducente de estos procedimientos en el mediano y largo plazo. De hecho, el poco prestigio del sistema judicial tiene que ver el déficit de eficacia moral de las normas legales y morales existentes.

¿Dónde buscar las fuentes de la eficacia moral? En la creación y en el ser escuchados. El primer elemento, central, es particularmente notorio en nuestro espacio social, aunque se lo ha visto únicamente desde su 'lado oscuro', valga el contrasentido: su escasa institucionalización. El reverso es un amplio espacio para el despliegue de diferentes formas de ingenio y adaptación que han producido un canon moral implícito que está en creciente contradicción con los enunciados públicos sobre las maneras tutelares de asegurar un orden público moralmente legítimo. Poder traducir en palabras este nuevo canon moral implícito es probablemente una de las principales tareas en la creación de una cultura pública alternativa.

El segundo elemento, la capacidad de ser escuchados solamente es posible en la medida que se reconozca la importancia pública de la conversación, del bullicio del ágora y del ejercicio de las argumentaciones. A diferencia del ordenado y sosegado diálogo platónico, aquí intervienen varias voces, también hay sonidos que vienen de los artefactos y poco a poco hay como una decantación de silencios progresivos que permiten la aparición de nuevos acordes y atender a un nuevo tema de conversación, debate, reclamo, según sea el caso. Las formas de acallar la conversación vienen por vías que no se reducen a la imposición dictatorial de un lenguaje político. No pocas veces la descalificación de la conversación ciudadana viene del lenguaje sofisticado de una tecnociencia que se presenta como ajena de los intereses y deseos humanos. Se trata de algo muy distinto a la tradicional comunicación vertical del maestro y el alumno o el lenguaje de la orden prepotente.

Una consecuencia es lo que podría llamarse el abandono de una 'retórica de la aceptación unánime'. Hablar acerca de una cuestión normativa como si 'todo el mundo' estuviera de acuerdo. Esa es la marca que deja el modelo ceremonial como fundamento moral. En el tiempo ceremonial el lugar y el momento están orientados a producir sentimientos unánimes. Ahí no hay lugar para la conversación ni para la diferencia de perspectivas o puntos de vista. Este modelo ceremonial ha tenido una amplia vigencia como escenario generador de normas morales. Una de las varias consecuencias nefastas ha sido la ya mencionada prioridad de la salvación por sobre las demandas de la justicia secular y la otra ha sido la hasta hace relativamente poco la condición de supralegalidad en la que movían sus miembros.

La retórica de la aceptación unánime es particularmente notoria en el caso 
de los clérigos católicos que hablan como si 'todo el mundo' fuera miembro de su iglesia, es una constante negación de cualquier perspectiva pluralista. Un discurso de estas características además, no hace más que fomentar y atraer temperamentos intolerantes y de una muy limitada eficacia para poner límites morales en las actividades cotidianas.

Luego del anterior recorrido que he presentado se puede apreciar que en el debate acerca del estado laico hay algunos términos que han cambiado. Si antes el problema fue el de la laicidad opuesta a un estado confesional, hoy el problema se plantea entre una laicidad, expresada en una moral de la invención, versus la autoridad tutelar. La línea divisoria entre los seguidores de alguna o ninguna religión pierde la relevancia anterior pues ahora ya no se trata principalmente de enfrentarse a definiciones doctrinarias religiosas y su aplicación en el estado. El período de destrucción-invención que hemos vivido, y que sigue vigente a escala global, ha introducido significativas novedades, la más importante es la aparición, con un perfil nítido, de la capacidad para enfrentar nuevos problemas con el despliegue de la imaginación, junto con la confianza que surge del trabajo bien hecho.

Con ello la tutela de la clase ociosa ${ }^{17}$ es cada vez menos necesaria. En otras palabras, la invención tiene como una de sus consecuencias la inhibición de los impulsos destructivos. Se entiende además que hablamos de una invención 'híbrida' en el sentido que la mejora tecnológica no da por supuesta la distinción entre naturaleza y cultura, por el contrario, tiende a desarrollar una perspectiva más integradora. Por cuestiones que ya no tienen que ver con una crisis política, vemos que la cuestión de la destrucción y la invención merodea las disputas medioambientales existentes en la relación entre minería y agricultura.

A manerade corolario, considero que estaoposición entreinvención ydestrucción es lo que predomina en los debates contemporáneos a escala global: vivimos en una era donde la revolución en la tecnología de las comunicaciones ha aumentado la intensidad en la formación de comunidades y ciertamente una intensidad en doble sentido: tanto para las solidaridades como para las enemistades más abismales. El otro factor es el cambio o superación de la relación naturaleza/sociedad donde el desarrollo de una perspectiva holística, relacional entre humanos y no humanos se abre paso cada vez con más fuerza. El esfuerzo por lograr un efectivo estado laico es parte de este esfuerzo más amplio.

17 En el sentido que Thorstein Veblen (1944) le daba al término, sectores improductivos que vivían de la ostentación ceremonial y el prestigio. 


\section{Referencias bibliográficas}

Biondi Juan y Zapata Eduardo (2006). La palabra permanente: verba manent, scripta volant: teoría y prácticas de la oralidad en el discurso social del Perú. Fondo Editorial del Congreso del Perú, Lima.

Brundage James (2000). La ley, el sexo y la sociedad cristiana en la Europa medieval, Fondo de Cultura Económica, México.

Canetti Elias (1982). Masa y Poder, Muchnik Editores, Barcelona.

Derrida Jacques, Adolfo Barberá, and Patricio Peñalver Gómez (1997). Fuerza De Ley: El Fundamento Mistico De La Autoridad, Tecnos, Madrid.

Duby Georges (1982). El caballero la mujer y el cura: el matrimonio en la Francia feudal, Taurus, Madrid.

Kant Inmanuel (2002). Fundamentación para una metafisica de las costumbres' (1785), Alianza Editorial; Madrid.

Montaigne Michel (2007). Los Ensayos, Ed. Acantilado, Barcelona, 2007.

Nugent Guillermo (2010). El Orden Tutelar. Formas de autoridad en América Latina, DESCO/CLACSO, Lima.

Nugent Guillermo (1996). El poder delgado : fusiones, lejanias y cercanias en el diseño cultural peruano, Fundación Friedrich Ebert, Lima.

Sennett Richard (2009). El Artesano Anagrama, Barcelona.

Veblen Thorstein (1944). Teoría de la clase ociosa Fondo de Cultura Económica, México.

Verbitsky Horacio (2007). Cristo Vence. Editorial Sudamerican, Buenos Aires.

Verbitsky Horacio (2006). Doble Juego: La Argentina Católica y Militar, Editorial Sudamericana, Buenos Aires.

Villanueva Salvatierra Susan (2013). "Del registro del matrimonio religioso al registro del matrimonio civil en el Perú" en Revista Académica del RENIEC Vol. $1 \mathrm{~N}^{\circ} 1$, Lima.

Whitehead Alfred North (1944). Modos De Pensamiento, Editorial Losada, Buenos Aires. 